\title{
Scalable and Privacy-preserving Design of On/Off-chain Smart Contracts
}

\author{
Chao Li, Balaji Palanisamy and Runhua Xu \\ School of Computing and Information, University of Pittsburgh, USA \\ \{chl205, bpalan, runhua.xu\}@pitt.edu
}

\begin{abstract}
The rise of smart contract systems such as Ethereum has resulted in a proliferation of blockchain-based decentralized applications including applications that store and manage a wide range of data. Current smart contracts are designed to be executed solely by miners and are revealed entirely on-chain, resulting in reduced scalability and privacy. In this paper, we discuss that scalability and privacy of smart contracts can be enhanced by splitting a given contract into an off-chain contract and an on-chain contract. Specifically, functions of the contract that involve high-cost computation or sensitive information can be split and included as the off-chain contract, that is signed and executed by only the interested participants. The proposed approach allows the participants to reach unanimous agreement off-chain when all of them are honest, allowing computing resources of miners to be saved and content of the off-chain contract to be hidden from the public. In case of a dispute caused by any dishonest participants, a signed copy of the offchain contract can be revealed so that a verified instance can be created to make miners enforce the true execution result. Thus, honest participants have the ability to redress and penalize any fraudulent or dishonest behavior, which incentivizes all participants to honestly follow the agreed off-chain contract. We discuss techniques for splitting a contract into a pair of on/off-chain contracts and propose a mechanism to address the challenges of handling dishonest participants in the system. Our implementation and evaluation of the proposed approach using an example smart contract demonstrate the effectiveness of the proposed approach in Ethereum.
\end{abstract}

\section{INTRODUCTION}

Creating trust among mutually distrustful participants without involving a trusted third party has been a challenge for several decades. The growth of Bitcoin [11] and the emerging follow-up cryptocurrencies have positioned blockchain as a promising solution for creating trust in a decentralized environment. Smart contracts expand the use of blockchains by allowing mutually distrustful participants to reach an agreement upon the execution results of complex contracts without a trusted third party. In leading smart contract systems such as Ethereum [19] and NEO [12], every single step of the decentralized computation is performed and verified by miners in the blockchain network, who are incentivized by the cryptocurrency rewards to behave honestly. This combination of decentralized computation and cryptocurrency-based incentives has led to the development of a large number of decentralized applications including applications that store and manage a wide range of data [15]. Ethereum [19], the first blockchain system that supports Turing-complete smart contracts, had a peak market cap of \$134 billion [3] in 2018.
The Smart Contracts market is estimated to grow at a CAGR of $32 \%$ during the period 2017 to 2023 [14].

Current smart contracts are designed to be executed solely by miners and are revealed entirely on-chain, resulting in reduced scalability and privacy. In this paper, we discuss that scalability and privacy of smart contracts can be enhanced by splitting a contract into two separate contracts: (1) an off-chain contract encapsulating functions of the whole contract that involve high-cost computation and/or distinguishable logic that may reveal private information about the participants; (2) an on-chain contract encapsulating the remaining low-cost/nonsensitive functions of the whole contract. Replacing the whole smart contract with the pair of on/off-chain contracts enables deploying only the on-chain contract onto the blockchain. This also allows saving the computing cost of running the off-chain contract by miners in the Ethereum blockchain network while the sensitive information involved in the off-chain contract can be hidden from the public.

After generating the pair of on/off-chain contracts from the whole contract, when all the participants are honest, they can reach unanimous agreement on the result of off-chain contract, just as if the off-chain contract was deployed on-chain and executed by miners. However, if there is a dispute of the off-chain result, such as a dishonest participant trying to lie about it, it would be necessary to allow honest participants to resolve the dispute on-chain. In this paper, we propose an effective mechanism that allows any honest participant to leverage the already deployed on-chain contract to create an on-chain instance of the off-chain contract. The process of creating a new contract from an existing contract can establish a unique connection between the two contracts. Through this connection, only the off-chain contract agreed and signed by all the participants can pass the integrity verification and can be created by the on-chain contract as a verified instance. Then, only the execution result of the verified instance can enforce the state change of the on-chain contract. Thus, honest participants have the ability to redress and penalize any fraudulent or dishonest behavior, which incentivizes all participants to honestly follow the agreed off-chain contract. We implement and evaluate the proposed approach in the Ethereum official test network Kovan [7] with an example smart contract using Solidity [16]. Our implementation demonstrates the effectiveness of the proposed approach of using on/off-chain contracts in Ethereum. 


\section{ON/OFF-CHAIN SMART CONTRACTS}

In this section, we first introduce the creation and execution of a general smart contract in Ethereum. We then present the techniques for splitting a given smart contract into a pair of on/off-chain contracts and explain how the pair of contracts can be executed in Ethereum.

\section{A. Smart contracts in Ethereum}

There are two types of accounts in Ethereum, namely External Owned Accounts (EOAs) controlled by private keys owned by users and Contract Accounts (CAs) assigned to smart contracts. A user of Ethereum should first create a EOA with a pair of keys and then deploy smart contracts from the EOA, resulting in the creation of CAs associated with the smart contracts. A smart contract in Ethereum refers to a piece of program code that usually consists of multiple functions, a few parameters and perhaps some modifiers. After programming a smart contract in a language such as Solidity [16], a user can compile the contract to get its bytecode and Application Binary Interface $(A B I)$ and can send a contract creation transaction to the Ethereum network with bytecode and (optional) ABI. Upon receiving the transaction, miners will include the bytecode into the next block, meaning that a new smart contract has been created, whose CA can be deterministically computed from the address of its creator and a nonce. Each CA can be viewed as a small decentralized server that can act based on the functions in its contract and can store data (e.g., cryptocurrency) allowed by its contract. However, CAs are passive, meaning that execution of any function of deployed smart contracts must be invoked through either transactions sent by EOAs or messages sent from CAs. As a result, the transactions/messages, as well as function inputs inside them, are all recorded by the Ethereum blockchain, which makes the function outputs deterministic because all miners can execute the function with the same inputs and gets the same outputs. It is worth noting that one needs to pay Gas [19] for either deploying a new smart contract or calling a function of existing smart contracts in Ethereum. Gas can be exchanged with Ether, the cryptocurrency used in Ethereum, and Ether can be exchanged with real money.

As discussed above, Ethereum requests each invoked function of deployed on-chain contracts to be executed by all the miners for the purpose of getting trustworthy outputs in the decentralized environment. However, this all-on-chain execution model of smart contracts may drain the scarce network resources and introduce privacy risks, when the invoked function includes high-cost calculation or sensitive information. Therefore, instead of deploying a whole contract with all function included, we propose to split such problem functions from the whole contract to execute them off-chain while still executing the rest of functions on-chain. We present the technique of implementing this approach by splitting a given contract into a pair of on/off-chain contracts.

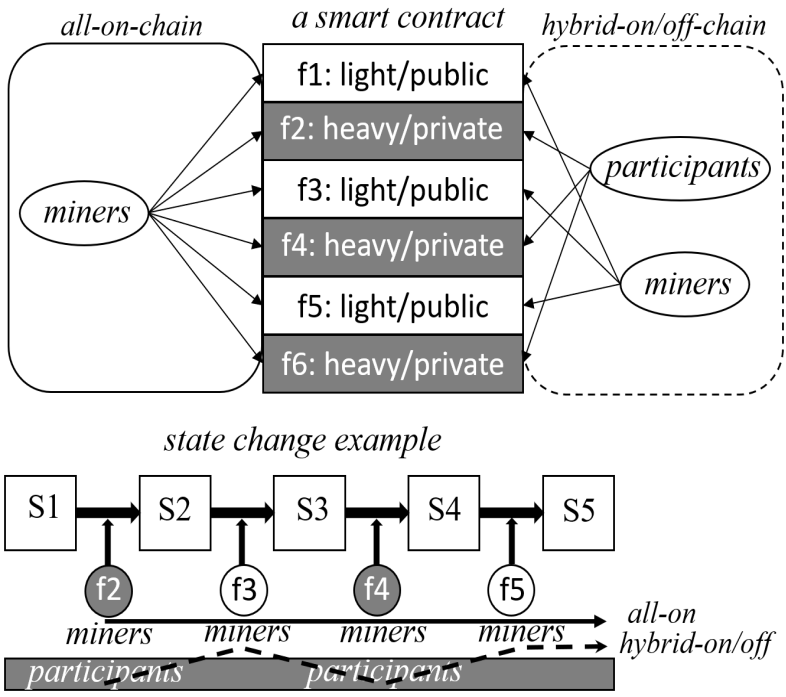

Fig. 1: Comparison of the current all-on-chain execution model of smart contracts in Ethereum and the proposed hybrid-on/off-chain execution model

\section{B. On/off-chain smart contracts}

We broadly classify functions of smart contracts into two categories based on the computing resources spent for executing them and the sensitive information carried by them: (1) heavy/private functions involving high-cost calculation or sensitive information and (2) light/public functions involving none of these features. For example, Alice and Bob may decide to bet on a private topic between them using cryptocurrency they have, so they draft a simplified betting contract as shown in Algorithm 1, which consists of three functions. They can first make deposits to the contract through deposit(), then invoke reveal() after a certain temporal threshold to reveal the result and finally reassign the cryptocurrency locked in the contract based on the result by calling reassign(). Since both deposit() and reassign() are simple cryptocurrency transfer functions, they contain neither high-cost calculation nor sensitive information, so they can be allocated to light/public functions. In contrast, reveal() may contain details of the customized betting rules that are private to the participants and may involve an arbitrary amount of computational cost, hence it should be allocated to a heavy/private function. It could be quite customized to allocate functions into the two categories. Here we recommend to allocate all functions of cryptocurrency transfer into light/public functions and consider the remaining ones as heavy/private functions.

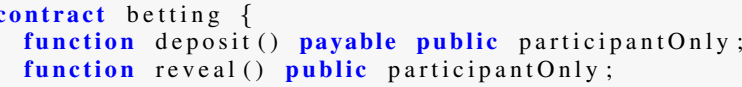

Algorithm 1: A simplified betting contract

After all functions of a smart contract has been classified, the heavy/private functions and light/public functions can be grouped into an off-chain contract and an on-chain contract, 
respectively. Then, only the on-chain contract needs to be deployed to be publicly executed by all the miners while the off-chain contract can be privately executed by only a small group of interested participants. Ideally, splitting the off-chain contract from the whole contract does not affect the state change of the on-chain contract. For clarity of illustration, consider an example smart contract in Fig. 1. which consists of three light/public functions (i.e., $c 1, c 3, c 5$ ) and three heavy/private functions (i.e., $c 2, c 4, c 6$ ). With the all-on-chain model, the whole contract consisting of all the six functions is deployed and miners need to execute function $f 2, f 3, f 4$ and $f 5$ to change the state of the deployed contract from $S 1$ to $S 5$. In contrast, with the hybrid-on/off-chain model, only an on-chain contract consisting of function $f 1, f 3$ and $f 5$ is deployed, so miners only need to execute function $f 3$ and $f 5$ while $f 2$ and $f 4$ can be privately executed by interested participants. As long as the participants are honest, they will be able to reach unanimous agreement on the off-chain execution results of $f 2$ and $f 4$ and input these results to turn the contract state from $S 1$ to $S 2$ and later from $S 3$ to $S 4$, just as if both $f 2$ and $f 4$ were executed by miners on-chain. However, in case of dishonest participants trying to lie about the off-chain execution results, we need an additional mechanism to always allow honest participants to enforce the true execution results of the off-chain contract. We will introduce this mechanism in the next section.

\section{ENFORCING OFF-CHAIN CONTRACTS}

Our proposed enforcement mechanism is used by honest participants to enforce the results of off-chain execution and penalize any dishonest participants.

In the presence of any dishonest participants, an honest participant can simply deploy the heavy/private functions back to the blockchain, make them get recomputed by the miners to enforce the state of on-chain contract to be changed as expected. However, there are two challenges to make this strategy work as expected. First, the honest participant must prove that the deployed heavy/private functions are exactly same as the original ones agreed by all the participants at the beginning. Otherwise, participants can falsify these functions based on their self-interests. Second, after verifying the integrity of the deployed heavy/private functions, we need to rebuild a connection between these functions and the already deployed light/public functions (i.e., on-chain contract). Since the two groups of functions have been separated from a single smart contract, such a connection is necessary to make them re-recognize each other, just as if they were deployed together in a single smart contract at the beginning.

To overcome the aforementioned challenges altogether, in Fig 2, we propose a general mechanism of enforcing off-chain contracts in the hybrid-on/off-chain execution model of smart contracts, which consists of four stages:

- Split/generate: Before any on-chain activity, the original contract is first split to two parts that package light/public functions and heavy/private functions, respectively. Obviously, the two parts themselves usually do not contain any

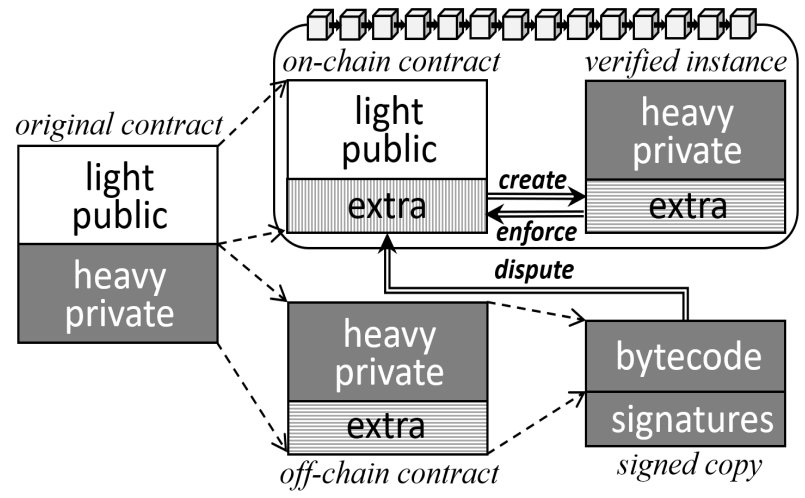

Fig. 2: Two categories of functions in the whole contract are padded with a few extra functions to form the on-chain contract and the off-chain contract. In the presence of dishonest participants, any honest participant can use the signed copy of the off-chain contract to make the on-chain contract create a verified instance of the offchain contract and leverage the unique link between the instance and its creator (i.e., on-chain contract) to enforce state change of the onchain contract.

function for resolving a dispute, so we need to pad each group of functions with a few extra functions prepared for a dispute. After padding, we have already generated an onchain contract as well as another off-chain contract from the whole contract.

- Deploy/sign: The on-chain contract (i.e., light/public functions including the padded extra functions) can then be deployed by any participant to the blockchain while the off-chain contract (i.e., heavy/private functions plus padded extra functions) needs to be converted into bytecode to be signed by all the participants. It is important to note that each participant must obtain a copy of the off-chain contract with signatures from all the participants before any interaction with the deployed on-chain contract can take place. The procedure of generating signed copies may easily be implemented through off-chain communication approaches, such as Whisper [18] in Ethereum.

- Submit/challenge: When all participants are honest, they can execute computation of the off-chain contract by themselves and manually submit the results to the on-chain contract to push the state change. This can be implemented by leaving a challenge period after a representative of the participants have submitted the result, during which all other participants can challenge the result with the signed copy of the offchain contract. Obviously, if the representative is honest, there will be no need to challenge the submitted result, so the state of the on-chain contract can be successfully changed as expected without revealing any information of the offchain contract and also without requiring miners to perform the computation of heavy/private functions.

- Dispute/resolve: In the presence of any dishonest participants, such as if the representative violates the agreement, a dispute occurs. To resolve the dispute, during the challenge period, any honest participant can submit the signed copy, namely the bytecode and signatures together, to the onchain contract, where an extra function padded to the on- 


\section{Participants Alice and Bob.}

\section{Betting Rules}

\section{Rules:}

1. Before time $T_{0}$, Alice should deploy on-chain contract. Both the participants should keep a signed copy of the off-chain contract.

2. Before time $T_{1}$, both the participants can make a deposit (1 Ether) to on-chain contract through deposit() or request a refund through refundRoundOne().

3. Between $T_{1}$ and $T_{2}$, if either Alice's balance or Bob's balance in on-chain contract is not 1 Ether, the participants can request a refund through refundRoundTwo().

4. The time point $T_{2}$ is the temporal threshold when the betting result becomes available. After that, between $T_{2}$ and $T_{3}$, the participants can compute the result off-chain. The loser (say Alice) can then call reassign() to make on-chain contract transfer 2 Ether to winner's account.

5. Finally, if the loser refused to implement step 4, the game will go to dispute/resolve stage. After $T_{3}$, the winner (say Bob) can call the extra function deployVerifiedInstance() with the signed copy at the on-chain contract and then call the extra function returnDisputeResolution() at the verified instance to enforce the dispute resolution.

TABLE I: Betting rules

chain contract during the split/generate stage will be called to verify the signed copy through signatures and create a verified on-chain instance. The deployed verified instance is created through the bytecode of the off-chain contract, so it is cloned from the off-chain contract and consists of all the heavy/private functions and padded extra functions in the off-chain contract. A participant (may or may not be the one submitting the signed copy) can then invoke the heavy/private functions within the verified instance to make them be executed by the miners. The execution result will then be the true one, which is different from the false result submitted by the dishonest participant during the dispute/resolve stage. Then, an extra function padded to the off-chain contract will be invoked to send the true result back to the on-chain contract and another extra function padded to the on-chain contract will receive the true result, enforce the dispute resolution and penalize the dishonest participant. To sum up, in the fourth stage, three extra functions are designed to resolve a dispute:

- deployVerifiedInstance(): We design this extra function at the on-chain contract to receive the signed copy during the challenge period. Once called, this function first verifies all the received signatures and then creates an on-chain instance for the bytecode within a signed copy that has passed the verification.

- returnDisputeResolution(): We design this extra function at the off-chain contract to return the true result computed by miners to the on-chain contract.

- enforceDisputeResolution(): We design this extra function at the on-chain contract to enforce the dispute resolution.

With this four-stage mechanism, honest participants always keep the ability to make dishonest participants be verified and monetary penalized, which in turn incentivizes all participants to honestly follow the results of off-chain smart contracts.
Next, we design and implement the proposed approaches using a detailed example.

\section{IMPLEMENTATION}

We implement the proposed approaches using an example smart contract for betting between Alice and Bob. The betting rules in the example smart contract are listed in Table [ Note that the presented betting rules do not involve penalty to dishonest participants, however it is straight-forward to revise step 2, 3 and 4 to monetarily penalize any dishonest participant in step 5 .

The contracts are programmed with Solidity and the offchain signature part is implemented using JavaScript with ethereumjs-util [4] package and web3-utils [17] package. All the contracts have been tested over the Ethereum official test network Kovan [7].

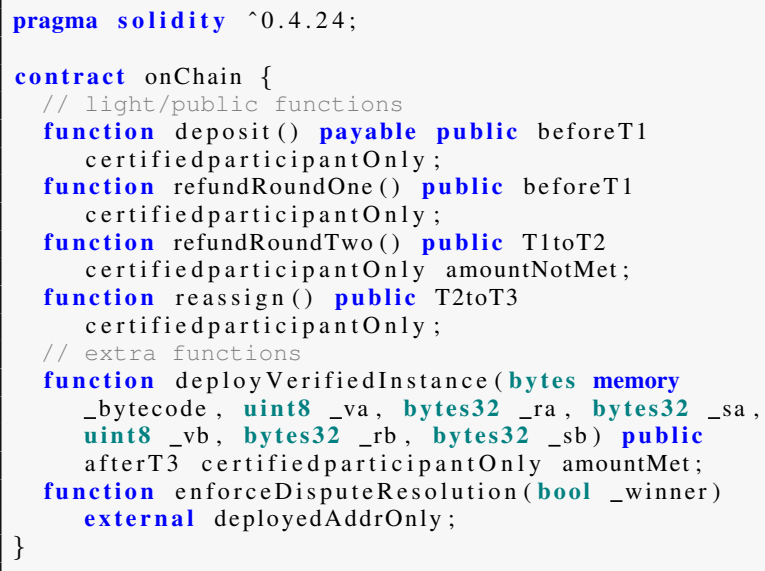

Algorithm 2: on-chain contract (interface)

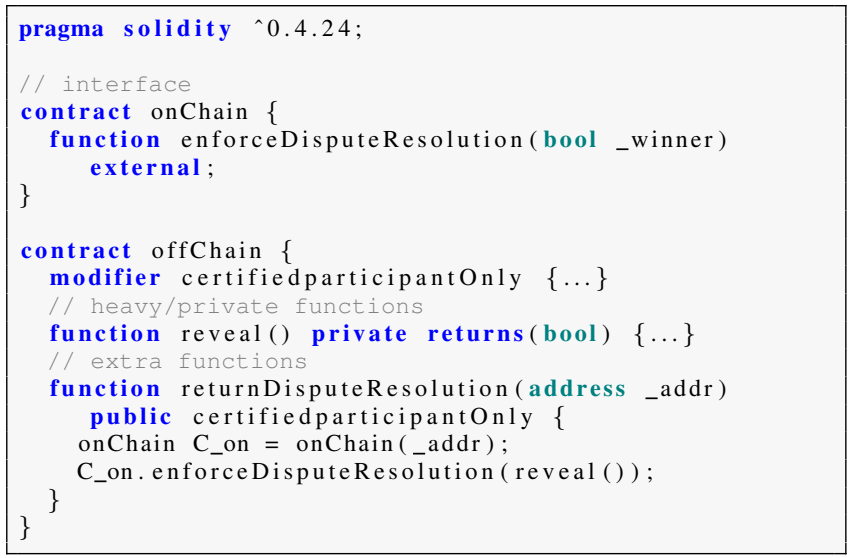

Algorithm 3: off-chain contract (simplified)

In rule 1 , before $T_{0}$, the on-chain contract should be deployed and the participants should have obtained signed copy of the off-chain contract. We show the implementation of the two contracts with Solidity in Algorithm 2 and Algorithm 3 respectively. As can be seen, in this example, the onchain contract consists of four light/public functions and two extra functions while the off-chain contract consists of one heavy/private function and one extra function. Here we note 
that the parameters, constructor and modifiers (e.g., beforeT1, certifiedparticipantOnly) are omitted in Algorithm 2 .

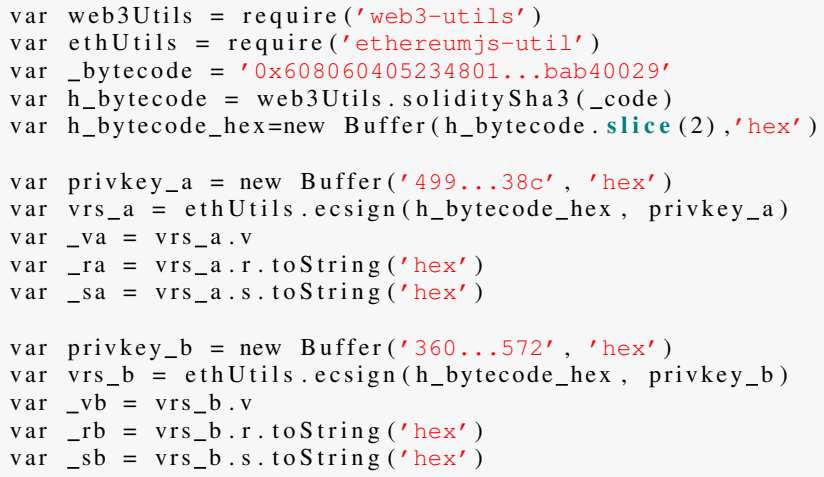

Algorithm 4: Signature v-r-s generation

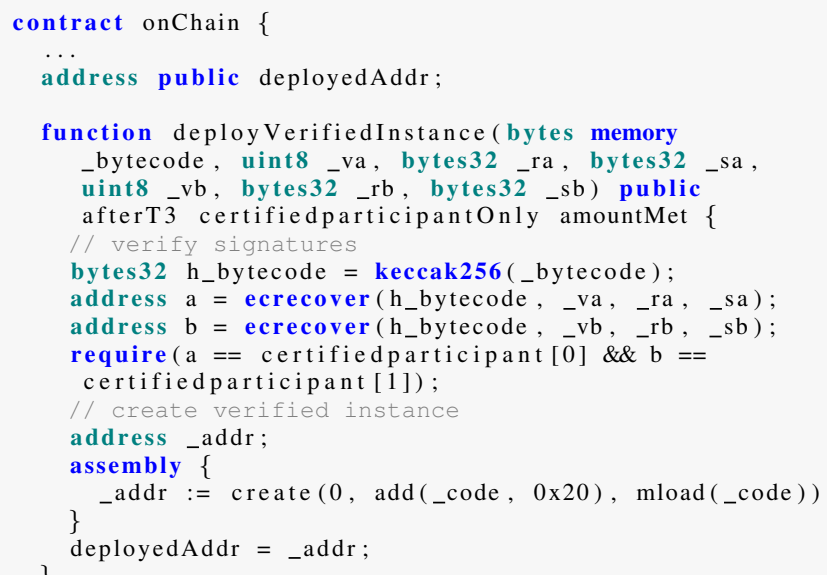

Algorithm 5: deployVerifiedInstance()

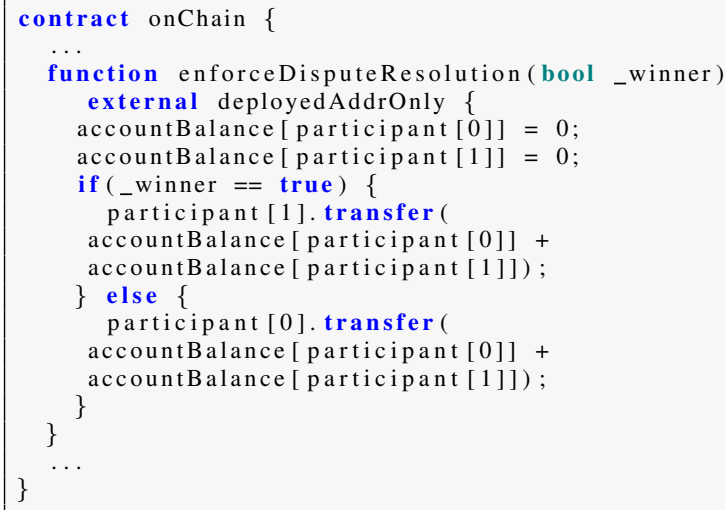

Algorithm 6: enforceDisputeResolution()

After generating the two contracts, Alice and Bob should transform the off-chain contract shown in Algorithm 2 into bytecode through tools such as Remix or Truffle and then use the JavaScript program in Algorithm 4 to generate signatures (tuples of $(v, r, s)$ ) with their account private keys and the bytecode. Please note that all the participants should use the same version of compiler for the purpose of getting same bytecode.

In case if Alice or Bob violates the rules before $T_{2}$, the game can always be terminated through refundRoundOne() or refundRoundTwo(). After $T_{2}$, suppose Alice loses the game, she should call reassign() to admit this failure before $T_{3}$ to let the function transfer both winner's deposit and loser's deposit to winner. If reassign() is not called before $T_{3}$, the winner will find the fact that the loser has violated the rules and a dispute has occurred. Once the dispute happens, after $T_{3}$, the winner (say, Bob in this example) should call the deployVerifiedInstance() extra function (Algorithm 5) with the signed copy. The extra function will first verify the signatures by outputting an address from bypecode and $(v, r, s)$ and checking whether it is the signer's address. After that, the extra function will create a verified instance from the bytecode and also record the address of the created instance in the parameter deployedAddr. Thus, by generating the verified instance from the on-chain contract, the verified instance can be authorized through the parameter deployedAddr because no other contract can have the same address and it is also guaranteed that the verified instance has been agreed by all the participants. Please note that to create a contract from an existing contract with only bytecode, we must use the assembly language in Solidty.

Finally, the winner (i.e., Bob) can call returnDisputeResolution() in the verified instance (see Algorithm 3) with the address of the on-chain contract (see Algorithm 2). The input address of the on-chain contract will then allow the verified instance to return the dispute resolution to the on-chain contract by invoking the extra function enforceDisputeResolution() (Algorithm 6, which then enforces both winner's deposit and loser's deposit to be transferred to winner and may also monetarily penalize the dishonest loser if there is a penalty rule. Here, the deployedAddrOnly modifier will check msg.sender so that only the verified instance with the address same as the one recorded in parameter deployedAddr can leverage enforceDisputeResolution() to change the state of onchain contract.

\begin{tabular}{|c|c|}
\hline Extra function & Gas cost \\
\hline deployVerifiedInstance() & $225082+$ reveal() \\
\hline returnDisputeResolution() & 37745 \\
\hline
\end{tabular}

TABLE II: Gas cost

We now present the gas cost for dispute resolution. As shown in Table II, in case of a dispute, an honest participant needs to first spend $(225082+$ cost of reveal ()$)$ gas to deploy the verified instance through deployVerifiedInstance() and then spend 37745 gas to enforce the resolution through returnDisputeResolution(). The overall cost is not high when cost of reveal() is low. However, if reveal() is a heavy function, it should be mandatory for each participant to pay security deposit so that the honest participant paying for dispute resolution can receive compensation from dishonest participants. 


\section{RELATED WORK}

Recently, off-chain resources of blockchain have been widely studied by researchers to improve the performance of blockchains [2], [5], [8], [9], [10], [13]. Among them, [5] and [10] are most relevant to our work. In [10], smart contracts are implemented using hybrid architectures similar to the hybrid-on/off-chain execution model proposed in our work. However, the proposed hybrid architectures in [10] rely on a Trusted Third Party (TTP) as an oracle and thus the architectures are not completely decentralized. In [5], a smart contract system named Arbitrum is developed, where smart contracts are designed to be executed off-chain. As the system is specially designed for this purpose, it is hard to generalize the system-level design to existing systems such as Ethereum. In this paper, instead of treating the use of offchain contracts as a system-level design goal, we consider the hybrid-on/off-chain computation model as an applicationlevel smart contract design pattern and also as a building block for enhancing blockchain scalability and privacy. Thus, the proposed approach is a plug-and-play solution that is compatible with existing smart contract systems and their time-tested infrastructure and community. In addition, the combination of the proposed approach and other system-level or application-level solutions, such as sharding [1] and zero knowledge proof [6], can further enhance the scalability and privacy of the smart contract systems.

\section{CONCLUSION}

In this paper, we propose a general hybrid-on/off-chain execution model of smart contracts, which separates the heavy/private functions of a smart contract from the light/public ones to form an off-chain contract and enables the off-chain contract to be executed only by the interested participants. The proposed approach leads to increased saving of computing resources of the miners and protects sensitive information of a smart contract in the blockchain. To handle disputes caused by any dishonest participants, we propose a mechanism that allows any honest participant to reveal a signed copy of the off-chain contract so that a verified instance can be created to make miners enforce the true execution result. Therefore, with the proposed approach, honest participants always have the ability to redress and penalize any fraudulent or dishonest behavior, which incentivizes all participants to honestly follow the agreed off-chain contract. Our implementation and evaluation of the proposed approach using an example smart contract demonstrate the effectiveness of the proposed approach of using on/off-chain contracts in Ethereum.

\section{REFERENCE}

[1] Mustafa Al-Bassam, Alberto Sonnino, Shehar Bano, Dave Hrycyszyn, and George Danezis. Chainspace: A sharded smart contracts platform. arXiv preprint arXiv:1708.03778, 2017.

[2] Jacob Eberhardt and Stefan Tai. On or off the blockchain? insights on off-chaining computation and data. In European Conference on ServiceOriented and Cloud Computing, pages 3-15. Springer, 2017.

[3] Ethereum market cap. https://coinmarketcap.com/currencies/ethereum/

[4] ethereumjs-util. https://github.com/ethereumjs/ethereumjs-util
[5] Harry Kalodner, Steven Goldfeder, Xiaoqi Chen, S Matthew Weinberg, and Edward W Felten. Arbitrum: Scalable, private smart contracts. In Proceedings of the 27th USENIX Conference on Security Symposium, pages 1353-1370. USENIX Association, 2018.

[6] Ahmed Kosba, Andrew Miller, Elaine Shi, Zikai Wen, and Charalampos Papamanthou. Hawk: The blockchain model of cryptography and privacy-preserving smart contracts. In 2016 IEEE symposium on security and privacy $(S P)$, pages 839-858. IEEE, 2016.

[7] Kovan: Ethereum official testnet. https://kovan.etherscan.io/

[8] Chao Li and Balaji Palanisamy. Decentralized privacy-preserving timed execution in blockchain-based smart contract platforms. In 2018 IEEE 25th International Conference on High Performance Computing (HiPC), pages 265-274. IEEE, 2018.

[9] Chao Li and Balaji Palanisamy. Decentralized release of self-emerging data using smart contracts. In 2018 IEEE 37th Symposium on Reliable Distributed Systems (SRDS), pages 213-220. IEEE, 2018.

[10] Carlos Molina-Jimenez, Ioannis Sfyrakis, Ellis Solaiman, Irene $\mathrm{Ng}$, Meng Weng Wong, Alexis Chun, and Jon Crowcroft. Implementation of smart contracts using hybrid architectures with on and off-blockchain components. In 2018 IEEE 8th International Symposium on Cloud and Service Computing (SC2), pages 83-90. IEEE, 2018.

[11] Satoshi Nakamoto. Bitcoin: A peer-to-peer electronic cash system, 2008.

[12] Neo. https://neo.org/

[13] Joseph Poon and Thaddeus Dryja. The bitcoin lightning network: Scalable off-chain instant payments. See https://lightning. network/lightningnetwork-paper. pdf, 2016.

[14] Smart contracts market research report global forecast to 2023. https: //www.marketresearchfuture.com/reports/smart-contracts-market-4588

[15] State of the dapps. https://www.stateofthedapps.com/

[16] The solidity contract-oriented programming language. https://github. com/ethereum/solidity

[17] web3-utils. https://www.npmjs.com/package/web3-utils

[18] Whisper. https://github.com/ethereum/wiki/wiki/Whisper

[19] Gavin Wood. Ethereum: A secure decentralised generalised transaction ledger. Ethereum Project Yellow Paper, 151, 2014. 